\title{
Criminologie
}

\section{Le clinicien et la question de la " dangerosité " : à la croisée du social et de l'individuel}

\section{Yann Bogopolsky}

Volume 17, numéro 2, 1984

La dangerosité, un débat à poursuivre

URI : https://id.erudit.org/iderudit/017201ar

DOI : https://doi.org/10.7202/017201ar

Aller au sommaire du numéro

\section{Éditeur(s)}

Les Presses de l'Université de Montréal

ISSN

0316-0041 (imprimé)

1492-1367 (numérique)

Découvrir la revue

Citer cet article

Bogopolsky, Y. (1984). Le clinicien et la question de la " dangerosité " : à la croisée du social et de l’individuel. Criminologie, 17(2), 93-101.

https://doi.org/10.7202/017201ar
Résumé de l'article

The concept of "dangerosity" as applied to a single individual excludes other forms of social violence.

I) A "mythical" function which names what appears threatening.

II) An "instrumental" function which identifies the so-called Social Disease, legitimizes the violence of incarceration, and pursues the quest for a rationale. III) A "symbolic" function which points out dangerous individuals in order to set them apart.

The clinician becomes a prisoner of that notion. As an expert he is forced by the judicial process to restrict the psychic reality down to the responsibility and irresponsibility levels. The play-then moves on from the social to the individual scene. Within the institution, he must tell fantasies and reality apart while at the same time - caught in the trap - he becomes the "watcher" of those excluded.

Society asks the clinician to restore its image of a perfect world but when the penalty is applied, it seems likely that it can be traced back to the same drives. 
LE CLINICIEN ET LA QUESTION DE LA «DANGEROSITÉ" À LA CROISÉE DU SOCIAL ET DE L'INDIVIDUEL Yann Bogopolsky*

The concept of "dangerosity" as applied to a single individual excludes other forms of social violence.

I) A "mythical" function which names what appears threatening.

II) An «instrumental" function which identifies the so-called Social Disease, legitimizes the violence of incarceration, and pursues the quest for a rationale.

III) A "symbolic" function which points out dangerous individuals in order to set them apart.

The clinician becomes a prisoner of that notion. As an expert he is forced by the judicial process to restrict the psychic reality down to the responsibility and irresponsibility levels. The play then moves on from the social to the individual scene.

Within the institution, he must tell fantasies and reality apart while at the same time - caught in the trap - he becomes the "watcher" of those excluded.

Society asks the clinician to restore its image of a perfect world but when the penalty is applied, it seems likely that it can be traced back to the same drives.

\section{INTRODUCTION}

Un tel flot d'écrits et de paroles fut déversé ces dernières années sur cette question controversée de la "dangerosité" qu'il semble aujourd'hui difficile de l'aborder sous un jour inédit... Et pourtant, par-delà cette abondance, nombreuses sont les zones d'ombre qui subsistent : comment déchiffrer le malaise éprouvé par certains cliniciens devant la persistance de son emprise? Comment rendre manifeste le miroir déformant à travers lequel on l'envisage? Comment saisir les glissements de sens qui inflitrent son utilișation?

Autant d'interrogations que nous tenterons de saisir à défaut de pouvoir les cerner.

\section{LE CLINICIEN DANS LA CITÉ}

Comme dans toute société, il y a certains risques auxquels nous nous exposons. Le droit d'être des citoyens libres est une option que nous choisissons volontairement au lieu de nous barricader dans des villas murées et d'enfermer en prison quiconque nous soupçonnons même de pouvoir nous nuire. Cela exige donc que nous soyons prêts à faire

\footnotetext{
* Criminoloque-psychotérapeute.
} 
face à la réalité de la violence qui existe et à chercher à y pourvoir directement (MacLatchie, 1984, p. 9).

Être singulier dans la société - comme tout citoyen - le clinicien jette un regard sur le monde qui l'entoure et dont il est partie prenante.

Que voit-il?

Il est le témoin d'une société qui accepte certains visages familiers de la mort (comme les pertes de vies humaines dans des accidents du travail ou de la circulation...) et sait tout autant se scandaliser de certaines de ses cuvres individuelles chez ceux qu'elle qualifie de meurtriers. Il est le spectateur d'une société qui tolère des enclaves de violence, tels les ghettos où préside la justice individuelle, telles ces tavernes très connues des bas-fonds des villes qui se veulent le théâtre de règlements de compte où se déroule un scénario tristement semblable à lui-même : une bagarre éclate désignant l'agresseur et l'agressé, ce dernier sort se procurer une arme et revient faire justice... Et ceci pour ne s'en tenir qu'à cette violence qui met aux prises les êtres humains dans leur réalité charnelle sans prendre garde aux activités frauduleuses qui, elles aussi, peuvent engendrer une autre forme de péril social.

Ainsi, le clinicien - en tant que membre du corps social - est-il connaisseur de divers contextes porteurs de violence qui ne semblent guère émouvoir la société et - en tant que professionnel - est-il acteur dans un nombre restreint de situations où l'emphase mise sur le danger n’a d'égal que le mythe qu'il suscite.

On attire l'attention sur le «danger» ou sur la violence individuelle plutôt que sur la violence collective ou structurelle. Ce sont les actes des individus plutôt que les conditions sociales ou les pratiques qui menacent encore plus sérieusement les biens que l'on dit vouloir protéger (la santé, la sécurité, la vie) qui sont perçus et définis comme «dangereux». (Landreville, Petrunick, 1981, p. 220).

C'est pourquoi la perspective qui confronte le clinicien à la notion de "dangerosité " ne peut être que réductrice du champ social de la violence.

Il n'est pas dans notre intention de réécrire ici, dans une perspective diachronique et critique, l'évolution de la notion de "dangerosité»; nombreux sont les auteurs qui nous ont précédés dans cette voie. Nous tenterons plutôt de cerner ce qui aujourd'hui encore en fait la persistance et semble s'inscrire dans un rapport de fonctions.

\section{A) FONCTION MYTHIQUE}

Cet acte ponctuel de mutilation ou de suppression d'une vie humaine, renvoie à des fantasmes ancrés dans l'inconscient collectif; fan- 
tasmes tels, qu'il semble nécessaire aux hommes de loi de mettre l'accent sur la concrétude des gestes accomplis, la virtualité des actes redoutés et la finitude temporelle de la punition à imposer; bref, de réinscrire l'acte dans la réalité et dans une codification. C'est ce que fit la Cour Suprême de l'État du New Jersey (États-Unis) en précisant :

Le comportement dangereux n'est pas identique au comportement criminel. La conduite dangereuse implique non seulement la violation des normes sociales qui sont imposées par des sanctions pénales, mais un dommage physique ou psychologique grave à des personnes ou une destruction substantielle de propriété. Les personnes ne devraient pas être incarcérées indéfiniment parce qu'elles présentent simplement des risques d'un futur comportement socialement indésirable (Cité par Montandon, C., 1979, p. 91).

Sans doute, de telles considérations devraient paraître élémentaires, mais le fait précisément qu'elles échappent à l'univers rationnel de ceux qui les manient révèle la base cachée sur laquelle cette notion repose. Ce caractère mythique de la "dangerosité" nous semble avoir été très bien cerné par J.D. André (1979, p. 224) lorsqu'il écrit : «le mythe est tout à la fois réaliste et onirique : réaliste, car la mort d'un gendarme en constitue la base objective; onirique, dans la mesure où l'angoisse, les fantasmes, les désirs incarnés dans le scénario, le poussent à exister (...). Le mythe a transmis (...), tel un révélateur, ces fantasmes qui serviront désormais de cadre d'interprétation future".

Ainsi, le mythe de la «dangerosité" — dans ces fantasmes qu'il vient interpeller - laisse émerger l'insécurité que le groupe social relie à la violence manifeste et éclatante de quelques êtres humains - nos semblables - et vient susciter une mise en acte dramatique qui deviendra cadre de compréhension. Cette «dangerosité», dont certains hommes deviennent les portefaix, acquiert le statut de prototype d'une destructivité susceptible de faire éclater le corps social; c'est pourquoi, la société pour se protéger de ce qui lui apparaît une menace pour son intégrité «a codifié le soupçon et le repérage des individus dangereux" (Foucault, M., 1982, p. 421 ).

\section{B) UNE FONCTION INSTRUMENTALE}

L'acquisition d'un corpus théorique et le nombre toujours croissant de critiques épistémologiques relatives à cette notion ne semblent guère entraver sa circulation qui se poursuit avec une vitalité égale à la ferveur des auteurs qui la condamnent. Toutefois, ses aléas maintenant bien connus et résumés par des auteurs tels Poupart, Dozois et Lalonde 
$(1982)^{1}$ dans leur article intitulé "L'expertise de la dangerosité» n'empêchent en rien son inscription dans la pratique criminologique, ni son emprise comme référant instrumental. En effet, cette notion de "dangerosité" nous apparaît tenir le rôle d'instrument polyvalent dont les fonctions manifestes sont de :

1) nommer le mal social pour le circonscrire et l'enclaver, pensant que cet enfermement protégerait à jamais la société des dangers de violence qui sont pourtant inhérents à sa structure ${ }^{2}$;

2) légitimer la violence de l'enfermement et de la déshumanisation, en réponse à l'acte violent posé par un être humain qui vient battre en brêche l'intégrité du corps social ${ }^{3}$;

3) pérénniser la quête illusoire du désir d'objectivité qui tiendrait lieu de vérité tout en réduisant l'être humain à une somme d'éléments dissécables et réifiables.

Il n'est qu'à prendre pour témoin les efforts incessants pour outrepasser les limites de nos connaissances actuelles relatives à la prédiction de la "dangerosité», comme nous en informe Monahan (1984) dans son dernier article sur "La seconde génération de penseurs" qui tiennent à relever le flambeau ${ }^{4}$ :

- en précisant que la faible fiabilité des données actuelles sur la violence reliée d'une part à la nature des prédictions (cliniques), d'autre part à la forme du cadre de recherche (emprisonnement à long terme) ne doit nullement conduire à l'évidence de l'absence d'une telle validité;

- en mettant de l'avant la possibilité d'amélioration de l'exactitude d'une telle prédiction par l'utilisation de nouvelles échelles;

- en prenant en compte des politiques qui reconnaîtraient le caractère relatif de telles prédictions.

Un tel regain de vitalité dans la recherche d'une plus grande fiabilité de la fonction instrumentale de la notion ne fait, selon nous, que renvoyer le débat sur un autre plan, celui de la philosophie du droit et de la fonction paradigmatique de la notion de «dangerosité».

1. Voir également Dozois, Lalonde et Poupart, 1982.

2. Cette vision relève de l'imaginaire puisque la société carcérale elle-même recrée ce mécanisme d'exorcisme en nommant de nouveaux bannis à l'intérieur même de ce lieu d'exclusion...

3. Sur la dangerosité en milieu pénitentiaire, voir François, 1981.

4. Voir également Monahan, 1983. 


\section{C) FONCTION PARADIGMATIQUE}

Être confronté à la notion de «dangerosité", c'est devoir s'interroger devant le non-sens apparent d'un acte. Ce non-sens peut glisser du côté de l'inexplicable (en ce qu'il échappe à la signification consciente) et de la folie ou peut s'arrimer à la loi; c'est dans cet ancrage à la loi que la notion de "dangerosité» nous semble jouer dans le champ criminologique un rôle symbolique équivalent à celui tenu par la notion de rétribution dans le domaine de la philosophie pénale.

Tout comme la peine constituerait «une sorte de procédure d'annulation de l'acte commis, qui doit restituer, dans son intégrité, la croyance ébranlée par le crime" (Poncela, 1983, p. 16), la notion de «dangerosité» acculée à une personne viendrait par son exclusion restituer l'intégrité bafouée du corps social. L'homme marqué du sceau de la "dangerosité» symbolise le crime à bannir, et la notion en elle-même devient le paradigme de ce qui doit être écarté pour la survie de la société.

\section{LE CLINICIEN ET L'EMPRISE DE LA «DANGEROSITÉ»}

$\mathrm{Qu}$ 'en est-il de la relation du clinicien à cet autre qui inscrit dans la rêalité événementielle des gestes qu'il serait aisé de verser au compte de la folie... Cette relation qui pourrait d'emblée s'entendre dans le domaine.privé est projetée sur la scène sociale : on en attend une parole dont les conséquences sont loin d'être anodines. Qu'en est-il précisément de ce rapport qui s"instaure dans cette situation de rencontre où se transcrit une réalité intérieure; réalité émergeant sur une trame événementielle qui, au lieu de l'éclairer, vient souvent l'occulter (Labadie, 1979). C'est bien dans ce lieu d'interférence que nous essaierons de saisir ce qui est mis en scène par le clinicien ou par l'imaginaire collectif et qui donne un éclairage particulier à ce type de rencontre.

\section{A) LE CLINICIEN ET SON SAVOIR SUR LA "DANGEROSITÉ"}

Pour d'aucuns, l'expertise est un acte professionnel d'importance dont le rationnel s'appuie sur les acquis théoriques, le savoir psychiatrique ou criminologique. Pourtant, en tant que clinicien, il nous apparait qu'un tel exercice est porteur de dilemmes, voire de contradictions internes.

«Pouvoir faire une expertise suppose que soit possible de tenir un discours qui dise le vrai sur un individu constitué en objet de son savoir" (Chardin, D., 1981, p. 74). On peut dès lors s'interroger sur l'objet du savoir psychiatrique ou criminologique et les nombreux glissements qui s'opèrent entre l'exposition de ce savoir et les fins qu'il sert, le statut de 
neutralité scientifique qu'il semble détenir et la réalité de témoin d'une parole qui est celle du clinicien. L'expertise se prête également à une falsification en ce qu'elle donne lieu à la transposition d'un langage qui se veut saisir d'une historicité indivíduelle en des catégories judiciaires de responsabilité ou d'irresponsabilité que ne sont plus du domaine de l'expert. Elle sème la confusion en maintenant l'apparence d'une pratique clinique par des entretiens qui - s'ils ne peuvent être assimilés à des entretiens thérapeutiques - n'en provoquent pas moins des effets du même ordre et l'utilisation de tels énoncés à des fins judiciaires.

L'expertise participe à une réduction qui n'est pas sans conséquence négligeable pour les êtres qui la subissent; en effet, plutôt que d'énoncer la «dangerosité» sur la scène sociale où l'être humain est acteur, elle la place par sa recherche étiologique, dans l'indi vidu qui se transforme alors en objet de maîtrise; la problématique de la "dangerosité» ne peut se comprendre sans la dimension de tolérance et son corollaire, l'exclusion, que viennent d'ailleurs sanctionner les représentants de la société lorsque le seuil en est outrepassé. Que le concept se déplace du champ de la psychiatrie à celui de la criminologie n'enlève rien à la pertinence de la question; car ce n'est pas le changement de cadre nosographique qui peut évacuer la réduction qui s'opère en localisant la "dangerosité" dans un individu, tandis que "la notion de dangerosité n'existe que dans l'espace de la relation aux autres" (Chardin, D., 1981, p. 81).

Nous ne prétendons pas pouvoir répondre à de telles contradictions, mais il nous semble par ailleurs impossible de les évacuer du champ de notre pratique; d'autant plus que ces écrans, venant opacifier la problématique, ne sont pas étrangers à la relation thérapeutique elle-même.

\section{B) LA "DANGEROSITÉ" OU L'IMPOSSIBLE CLOISONNEMENT DU CLINICIEN}

Le clinicien peut être enserré dans maints cloisonnements qui annihilent le sens de sa pratique. Le plus englobant (et peut-être le plus insidieux) est la mutation du traitement en mesure pénale. Le cadre institutionnel peut certes venir stigmatiser le carrefour des demandes contradictoires où se situe le clinicien : I'institution attend du thérapeute qu'il soit un instrument de réhabilitation; le thérapeute ne peut se laisser enfermer dans une telle perspective utilitaire s'il veut être réceptif à une démarche qui ne peut s'ébaucher que dans le contrat individuel et l'écoute thérapeutique. Mais cette écoute, elle-même, peut être piégée par les messages implicites ou explicites qui émanent de l'individu dit dangereux : déversement de fantasmes de violence auquel le thérapeute doit tenir lieu de contenant sans lui-même être happé par la violence du discours qui transforme les mots en armes, les désirs en actes. Cette 
prégnance de la fantasmatique avec laquelle le thérapeute est aux prises le renvoie à son propre univers interne où l'intuition est le seul repère en butte à ceux qui demandent des preuves. Preuves dont la concrétude serait garante de la bonne gestion du danger, mais qui, en négatif, laisseraient entrevoir la peur qui circule, infiltrant en sourdine la relation. "Une psychothérapie n'est peut-être pas autre chose que cette gestion des dangers qui envahissent le patient au prix du danger qu'il nous fait alors courir» (Buffard, D., 1981, p. 188). Danger imaginaire ou danger réel? Combien de fois le thérapeute est-il aux prises avec une inquiétude angoissante dont l'emprise n'a d'égal que le pouvoir du fantasme... de ceux qui représentent le "danger" et qui font vaciller notre propre imaginaire; seul, l'espace entre le fantasme et la réalité permet la poursuite de la démarche thérapeutique.

Il est une autre chose qui peut également se refermer insidieusement sur le clinicien : la garantie de sauvegarde dont on le rend responsable. En effet, la société n'a de cesse de retrancher de son corps social ceux qu'elle désigne comme "mauvais objets» et qui deviennent boucémissaires de sa propre agressivité; dans cette perspective, il n'est que trop tentant de transformer le clinicien en gardien des exclus, et en devin auquel incomberait la mission impossible d'identifier leur devenir.

\section{C) LE CLINICIEN ET L'IMAGINAIRE COLLECTIF}

"Le danger d'un acte délinquant serait peut-être porportionnel aux sollicitations qu'il soulèverait en notre propre inconscient de pulsions plus ou moins mal refoulées (...) En condamnant les coupables, nous nous donnerions, bien plus que la sécurité d'un ordre social déterminé, la certitude d'être innoncents de semblables pulsions» (Cassiers, L., 1981, p. 155). Ainsi, la violence des réactions du groupe social face à l'homme dangereux serait révélatrice de l'intensité des pulsions sous-jacentes à ce groupe. Freud explique l'émergence du sentiment de menace chez les primitifs par la transgression d'un tabou qui n'est pas immédiatement réprimé. Du tabou au désir, il n'y a qu'un pas à franchir et «il arrive souvent que le châtiment fournit à ceux qui l'exécutent l'occasion de commettre à leur tour, sous le couvert de l'expiation, le même acte impur. C'est là un des principes fondamentaux du système pénal humain, et il découle naturellement de l'identité des désirs refoulés chez le criminel et chez ceux qui sont chargés de venger la société outragée" (Freud, 1912, p. 86).

Mais, la société fait la sourde oreille à de telles vérités; elle aime se concevoir comme un tout intègre, sans aspérité pour ternir son image. Et l'on peut se demander si l'une des demandes inconscientes du groupe 
social adressée au clinicien ne serait pas de venir réparer cette blessure que l'homme fou, dangereux vient lui infliger.

Il ya dans cette opiniâtreté à vouloir prédire la dangerosité d'un être — par-delà le but avoué du prix de la vie humaine - le désir secret d'une correspondance parfaite entre la vision idéale (pure construction de l'esprit) et les images que les scénarios de sa réalité lui renvoient.

Le thérapeute aurait pour tâche de "purifier» la société, de faire correspondre attente et réalisation, de supprimer le manque, de nier la faille, l'imperfection...

Par-delà les schémas conceptuels, les références théoriques, telles les données statistiques par exemple, il nous importait de mettre en lumière la valeur de l'acte criminologique : accepter d'être un expert $n$ 'est pas un acte anodin en soi, accepter de se frotter à cette dangereuse question de la "dangerosité" n'est pas innocent... Faut-il se battre comme certains pour l'abrogation de ces législations d'exception, faut-il refuser de se prononcer sur la question...?

Quelle que soit la position adoptée par chacun, il nous semble essentiel de ne pas opposer la muraille du savoir à "l'inquiétante étrangeté» que provoque en nous la folie, de ne pas nier à celui-là même qui pose des actes insensés la reconnaissance du sujet, de ne pas exorciser nos propres peurs par une sempiternelle exclusion de cet autre venu faire irruption dans le champ repérable de notre quotidien.

\section{RÉFÉRENCES}

ANDRÉ J.D. (1979), "Presse et justice pénale : des fantasmes à la réalité», Déviance et société, vol. $3, \mathrm{n}^{\mathrm{0}} 3$, p. 201-227

BUFFARD, S. (1981) "Est-il dangereux de se pencher?", in Ch. Debuyst (édit.), Dangerosité et justice pénale. Ambiguïté d'une pratique, Genève, Médecine et Hygiène, p. 177-189.

CASSIERS, L. (1981), "La dangerosité comme péril d'inexistence psychique. Réflexion psychanalytique", in Ch. Debuyst (édit.), Dangerosité et justice pénale. Ambiguité $d^{\prime}$ une pratique, Genève. Médecine et hygiène, p. 155-160.

CHARDIN, D., F. M.-J. JAUSSET, N. VACHER (1971), "L'expertise psychiatrique, lieu d'alternatives?" Pratique de la folie, Paris, Solim, p. 71-84.

DOZOIS, J., M. LALONDE, J. POUPART (1982), "La dangerosité : un dilemme sans issue? Réflexion à partir d'une recherche en cours", Déviance et société, vol. $5, \mathrm{n}^{\circ} 4$, p. 383-401.

FOUCAULT, M. (1981), "L'évolution de la notion d'individus dangereux dans la psychiatrie légale", Déviance et société, vol. 5, no 4, p. 403-422.

FRANÇOIS, J. (1981), «La dangerosité en milieu pénitentiaire. Opérateur social et réalité fusionnelle", in Ch. Debuyst (édit.), Dangerosité et justice pénale. Ambiguité d'une pratique, Genève, Médecine et Hygiène, p. 83-107. 
FREUD, S. (1912), Totem et Tabou. Paris, Payot, 1972.

LABADIE, J.M. (1979), «Le landau lâche», la Nouvelle revue de psychoanalyse, vol. 19, p. 245-256.

LANDREVILLE, P. et M. PETRUNICK (1981), "Le «délinquant dangereux" dans les législations nord-américaines", in Ch. Debuyst (édit.), Dangerosité et justice pénale. Ambiguitté d'une pratique, Genève, Médecine et Hygiène, p. 207-229.

MACLATCHIE, J.M. (1984), «Nouveau regard sur la dangerosité», Bulletin de la société canadienne pour la prévention du crime, vol. $14, \mathrm{n}^{\circ} 4$, p. 9.

MONAHAN, J. (1973), "Dangerous Offenders. A Critique of Kozol and al.»Crime and Delinquency, vol. 19, $\mathrm{n}^{\circ} 3$, p. 418-419.

MONAHAN, J. (1984), "The Prediction of Violent Behavior: Second Generation Thinking", American Journal of Psychiatry, vol. 141, n" 1, p. 10-15.

MONTANDON, C. (1979), «La dangerosité, revue de littérature anglo-saxonne", Déviance et société, vol. $3, \mathrm{n}^{01} 1$, p. 89-105.

PONCELA, P. (1983), «Éclipses et réapparitions de la rétribution en droit pénal", Rétribution et justice pénale. Travaux du centre de philosophie du droit, Paris, Presses universitaires de France, p. 11-19.

POUPART, J., J. DOZOIS, M. LALONDE (1982), "L'expertise de la dangerosité ", Criminologie, vol. $15, \mathrm{n}^{\circ} 2$, p. 7.27. 